\title{
A Virtual Reality Approach for Exploring Spatio-Temporal Urban Data
}

\author{
Jorge A. Wagner Filho \\ Federal University of Rio Grande do Sul \\ jawfilho@inf.ufrgs.br
}

\author{
Luciana Nedel \\ Federal University of Rio Grande do Sul \\ nedel@inf.ufrgs.br
}

\begin{abstract}
One of the biggest challenges in computing nowadays is to extract relevant information from ever-growing datasets. Applications such as smart cities, transportation planning, control of epidemics, and citizen engagement in public governance can heavily benefit from the analysis of large volumes of urban data. Despite advances in AI and Data Mining, sometimes they are not enough. Data visualization allows us to apply our human visual understanding capabilities and domain knowledge to this process, and to explore the data without necessarily knowing beforehand what information we are looking for. We hypothesize that immersive and stereoscopic Virtual Reality (VR) environments, coupled with natural embodied interaction, will better support the exploration of inherently three-dimensional spatio-temporal data representations. Through the expansion of an immersive technique we have recently proposed, and iterative user evaluations employing real-world datasets, we will investigate this hypothesis and identify the most efficient design choices for interaction and collaboration.
\end{abstract}

Index Terms-Space-time cube, Trajectory visualization, Immersive analytics.

\section{INTRODUCTION}

Understanding how objects' or people's positions vary over time and extracting meaningful patterns and insights from these data is a topic of growing interest for a wide range of stakeholders, from regular people planning their journeys to human geographers and decision-makers preparing for possible situations in the future or analyzing the past. Nowadays, GPS-enabled mobile devices, telecommunication networks [1], and even social networks [2] can easily collect large and detailed datasets recording people's positions over time, with applications in a broad range of domains, including urban planning, mobility optimization, sports analytics, and behavioral studies. The high complexity and heterogeneity of these data invariably require the integration of human perception and domain knowledge, coupled with automated data analysis. Nevertheless, visualizing critical spatio-temporal features and patterns in such datasets is still a challenge.

\section{Motivation}

2D map representations focus on the spatial nature of the data and, even with the aid of animations, make difficult the observation of features such as varying movement durations and speeds, stop durations and locations and meetings between

We acknowledge financial support from CNPq-Brazil and Global Affairs Canada. This study was financed in part by the Coordenao de Aperfeioamento de Pessoal de Nvel Superior - Brasil (CAPES) - Finance Code 001. different individuals (when they share the same position in time and space). 3D representations are an alternative to more adequately address the temporal nature of the data, for example by employing the perpendicular axis to represent the time component, resulting in a Space-Time Cube (STC).

Originally proposed by Hagerstrand [3], the STC has been revisited by Kraak [4] in the context of an extended interactive geovisualization environment, and later applied to diverse domains, such as event data [5], ship trajectories [6], airtraffic analysis [7] and mobile phone positioning data [8]. However, and similar to other 3D data representations, the STC suffers from well-known limitations in terms of perception and interaction when it is used in conventional desktop setups. These are a result of the difficulties in estimating distances and visual depths from monocular depth cues alone and the mismatch in terms of control between a 3D environment and a $2 \mathrm{D}$ interaction device such as a mouse, as well as of the challenges introduced by occlusion and visual clutter. This is even worse when taking into account that domain experts are typically not-and should not be required to be-trained in 3D manipulation.

\section{RELATED WORK}

Some initial efforts to implement immersive representations of movement data, such as the STC, have been reported by Theuns [9], using a VR HMD-based prototype, and Saenz et al. [10], using an Augmented Reality (AR) HMD. Moran et al. [11] also explored a VR approach for the visualization of geo-tagged Twitter posts originated from the MIT campus. Tweets were distributed in a virtual recreation of the campus, and the particular representation of each was determined by its content. In HoloMaps, real-time geo-tagged tweets and traffic information were also displayed in a 3D city model, but using Hololens [12]. In the GeoGate AR system, Ssin et al. [13] combined a 2D tabletop display with a 3D AR "hologram" to visualize trajectories in the maritime domain. Due to the limited field of view of current AR HMDs, the STC is shown at a small size and its position is controlled by moving a tangible device, which works as a spatial filter. GeoGate reduced errors in tasks where users had to correlate different data sources.

\section{Proposed Solution}

Our proposed research challenge is the design and evaluation of efficient immersive representations for spatio-temporal 
data. We hypothesize that Immersive Analytics [14] environments based on head-mounted displays (HMD), coupled with natural interaction, will better support the visual exploration of these highly complex 3D data, reducing reported steep learning curves and interaction difficulties [8]. Our approach is based on the improvement and extension of the VirtualDesk framework, and its combination with complementary exploration metaphors such as egocentric flying and physical walking. In VirtualDesk, proposed in our previous studies on the immersive visualization of multidimensional information [15], the data is rendered in small scale above a virtual reproduction of the analyst's real desk, enabling embodied and tangible interaction and providing strong stereopsis and proprioception cues. This approach has shown accuracy benefits over a desktop alternative for distance and density perception tasks, while adding little or no time overhead and not causing simulator sickness, so far a serious concern in immersive applications. Given the heterogeneous nature of urban data, different coordinated views must be provided, and these can also be positioned on the surface of the analyst's virtual desk. Our research follows an iterative design and testing approach, through user studies with expert and non-expert participants.

\section{Preliminary Results}

Initial results for spatial-temporal data obtained in collaboration with the School of Interactive Arts and Technology at Simon Fraser University, Canada, confirmed the benefits of immersive exploration for the user's qualitative experience, with much higher usability and user preferences, very low simulator sickness incidence, and a lower measured mental workload, resulting in a publication to be presented at IEEE VIS 2019, a premier venue for Data Visualization research:

- WAGNER FILHO, J. A.; STUERZLINGER, W.; NEDEL, L. Evaluating an Immersive Space-Time Cube Geovisualization for Intuitive Trajectory Data Exploration. IEEE Transactions on Visualization and Computer Graphics, 2019. (to appear, Qualis A1)

We have also collected experts' recommendations on system improvements, through an ongoing collaboration with the STEAMER (Spatio-temporal information, adaptability, multimedia and knowledge representation) group at the Laboratoire dInformatique de Grenoble, France. This feedback was reported in a recent journal publication at IEEE CG\&A, 1 out of 4 works selected for a Special Issue on Immersive Analytics.

- WAGNER FILHO, J. A.; FREITAS, C.M.D.S.; NEDEL, L. Comfortable Immersive Analytics with the VirtualDesk Metaphor. IEEE Computer Graphics and Applications, 39(3), 2019. (Qualis A2)

\section{CONCLUSION}

The next steps for our research include further detailed evaluations with domain experts and the application of our approach to real-world spatio-temporal datasets in different domains, such as urban mobility and public health. Different categories of data (e.g., event data, origin-destination data, GPS trajectories) will result in different design requirements, and will also demand the integration of automated analytical approaches in order to support efficient visual exploration of larger volumes of data. Taking into account the typical workflow of data analysts, we will also investigate different possibilities for interaction and especially collaboration (either collocated or remote) techniques, and their effects on the overall analytical performance.

We expect this research to result in a complete immersive analytical environment, supporting complementary data exploration metaphors and capable of assisting different sorts of stakeholders, including city authorities, urban planners and researchers, in decision making processes. Since it is based on the use of low cost HMDs, it will also be capable of engaging regular users on casual data exploration and citizen participation. More broadly, our evaluation of immersive representations for spatio-temporal data will also result in new guidelines for the construction of efficient Immersive Analytics applications [14] and the design of appropriate interaction and collaboration techniques for such contexts.

\section{REFERENCES}

[1] F. Calabrese, L. Ferrari, and V. D. Blondel, "Urban sensing using mobile phone network data: A survey of research," ACM Computing Surveys, vol. 47, no. 2, pp. 25:1-25:20, 2015.

[2] A. Noulas, S. Scellato, C. Mascolo, and M. Pontil, "An empirical study of geographic user activity patterns in foursquare," in Fifth International AAAI Conference on Weblogs and Social Media. AAAI, 2011.

[3] T. Hägerstraand, "What about people in regional science?" Papers in Regional Science, vol. 24, no. 1, pp. 7-24, 1970.

[4] M.-J. Kraak, "The space-time cube revisited from a geovisualization perspective," in Proc. 21st International Cartographic Conference. Citeseer, 2003, pp. 1988-1996.

[5] P. Gatalsky, N. Andrienko, and G. Andrienko, "Interactive analysis of event data using space-time cube," in International Conference on Information Visualisation (IV). IEEE, July 2004, pp. 145-152.

[6] N. Andrienko and G. Andrienko, "Visual analytics of movement: An overview of methods, tools and procedures," Information Visualization, vol. 12, no. 1, pp. 3-24, 2013.

[7] S. Buschmann, M. Trapp, and J. Döllner, "Animated visualization of spatial-temporal trajectory data for air-traffic analysis," The Visual Computer, vol. 32, no. 3, pp. 371-381, 2016.

[8] I. Kveladze, M.-J. Kraak, and C. P. Van Elzakker, "The space-time cube as part of a geovisual analytics environment to support the understanding of movement data," International Journal of Geographical Information Science, vol. 29, no. 11, pp. 2001-2016, 2015.

[9] J. Theuns, "Visualising origin-destination data with virtual reality: Functional prototypes and a framework for continued VR research at the itc faculty," B.S. Thesis, University of Twente, 2017.

[10] M. Saenz, A. Baigelenov, Y.-H. Hung, and P. Parsons, "Reexamining the cognitive utility of 3D visualizations using augmented reality holograms," in IEEE VIS Workshop on Immersive Analytics. IEEE, 2017.

[11] A. Moran, V. Gadepally, M. Hubbell, and J. Kepner, "Improving big data visual analytics with interactive virtual reality," in HPEC, 2015 IEEE. IEEE, 2015, pp. 1-6.

[12] R. Hills-Duty, "Taqtile are creating new holomaps for the hololens," https://www.vrfocus.com/2017/07/taqtile-are-creating-new-holomapsfor-the-hololens, 2017, accessed: 2017-10-22.

[13] S. Y. Ssin, J. A. Walsh, R. T. Smith, A. Cunningham, and B. H. Thomas, "Geogate: Correlating geo-temporal datasets using an augmented reality space-time cube and tangible interactions," in 26th IEEE Conference on Virtual Reality and 3D User Interfaces, March 2019.

[14] T. Chandler, M. Cordeil, T. Czauderna, T. Dwyer, J. Glowacki, C. Goncu, M. Klapperstueck, K. Klein, K. Marriott, F. Schreiber et al., "Immersive analytics," in 2015 BDVA. IEEE, 2015, pp. 1-8.

[15] J. A. Wagner Filho, C. M. Freitas, and L. Nedel, "VirtualDesk: A Comfortable and Efficient Immersive Information Visualization Approach," Computer Graphics Forum, vol. 37, no. 3, pp. 415-426, 2018. 